\title{
Evaluation du niveau de contamination des eaux souterraines par les éléments traces métalliques dans le département de Zouan-Hounien (Ouest de la Côte d'Ivoire).
}

Péhégninon Junior Ophélie DJADE1, Abou TRAORE11, Koffi Jean Thiérry KOFFI2, Keiba Noël KEUMEAN', Gbombélé SORO'1, Nagnin SORO'1

1 Université Félix Houphouet Boigny de Cococdy, BP 582 Abidjan 22 (Côte d'Ivoire): junorphee03@gmail.com

2 Université Nangui Abrogoua, 08 BP 109 Abidjan 08 (Côte d'Ivoire) : thierrykoffi2000@gmail.com

Original submitted in on $23^{\text {rd }}$ March 2020. Published online at www.m.elewa.org/journals/ on $30^{\text {th }}$ June 2020 https://doi.org/10.35759/JABs.150.6

\section{RESUME}

Objectif : Evaluer le niveau de contamination des eaux souterraines de Zouan-Hounien en élément traces métalliques (ETM).

Méthodologie et résultats: Un total de soixante-douze (72) échantillons d'eaux souterraines ont été prélevés en raison de quarante-six échantillons d'eaux de puits (23 puits) et vingt-six échantillons d'eaux de forages (13 forages). Dans ces échantillons, des ETM tels que : $\mathrm{Hg}, \mathrm{Pb}, \mathrm{Cd}$, As et Fe ont été dosés par spectrométrie d'absorption atomique. Les concentrations moyennes respectives de $\mathrm{Fe}, \mathrm{Pb}, \mathrm{Hg}, \mathrm{As}$ et $\mathrm{Cd}$ sont de 2233,48>3,10>1,67>1,18>0,08 $\mu \mathrm{g} \cdot \mathrm{L}^{-1}$ dans les puits et de 2427,94>4,08>, 2,36>1,76> $0,08 \mu \mathrm{g} \cdot \mathrm{L}^{-1}$ dans les forages. La valeur moyenne du degré de contamination (Dc) dans les puits et les forages est supérieure à 3 , indiquant ainsi une forte contamination des eaux souterraines. Avec des valeurs moyennes de l'indice de pollution par les ETM (HPI) inférieures à la valeur seuil de 100, ces eaux restent faiblement polluées dans l'ensemble. Pourtant, deux puits et deux forages ont enregistré une forte pollution des eaux, avec des valeurs supérieures à 100 .

Conclusion : L'indice de pollution des eaux souterraines par les ETM appliqué aux eaux souterraines révèle que les eaux de puits et de forages sont de bonne qualité, à l'exception de deux puits et deux forages. Le mercure reste le principal élément qui contribue à la toxicité des eaux. Sa présence dans les eaux est due à l'effet des activités d'orpaillage ancien et actuel dans la zone. Une sensibilisation sur les impacts de l'orpaillage sur les ressources en eau est à mener au sein des orpailleurs afin de réduire l'utilisation du mercure. Une consommation prolongée de ces eaux peut entrainer des problèmes graves de santé publique.

Mots clés : indices de pollution, eaux souterraines, ETM, orpaillage, Zouan-Hounien 
Assessment of pollution indices by metallic trace elements of groundwater resources in the mining area of the department of Zouan-Hounien, Côte d'Ivoire.

Objective : Assess the level of contamination of Zouan-Hounien groundwater with metallic trace elements (ETM).

Methodology and results: A total of seventy-two (72) groundwater samples were taken that is forty-six well water samples ( 23 wells) and twenty-six borehole water samples ( 13 wells). In these samples, ETMs such as: $\mathrm{Hg}, \mathrm{Pb}, \mathrm{Cd}, \mathrm{As}$ and $\mathrm{Fe}$ were determined by atomic absorption spectrometry. The respective average concentrations of $\mathrm{Fe}, \mathrm{Pb}, \mathrm{Hg}, \mathrm{As}$ and $\mathrm{Cd}$ are 2233.48>3.10>1.67>1.18>0.08 $\mu \mathrm{gg} \mathrm{L}^{-1}$ in the wells and $2427.94>4,08>, 2.36>1.76>0.08 \mu \mathrm{g} . \mathrm{L}^{-1}$ in boreholes. The average value of the degree of contamination (Dc) in wells and boreholes is greater than 3 , thus indicating a strong contamination of groundwater. With average values of the ETM pollution index (HPI) below the threshold value of 100 , these waters remain slightly polluted. However, two wells and two boreholes recorded heavy water pollution, with values greater than 100. The correlation matrix carried out between the ETM and the HPI reveals that $\mathrm{Hg}$ is the main element, which contributes to the toxicity of the water.

Conclusion: The ETM pollution indices for groundwater applied to waters revealed that well and borehole water are of good quality, with the exception of two wells and two boreholes. However, mercury remains the main element that contributes to the toxicity of water. Its presence in the waters is due to the effect of old and current gold panning activities in the area. Thus, raising awareness of the impacts of gold panning on water resources is to be carried out among gold panners in order to reduce the use of mercury.

Prolonged consumption of these waters can lead to serious public health problems.

Keywords: pollution indices, groundwater, ETM, gold panning, Zouan-Hounien

\section{INTRODUCTION}

La contamination des eaux souterraines par les éléments traces métalliques (ETM) reste aujourd'hui, l'un des problèmes environnementaux les plus importants du monde (Vodela et al., 1997). Les métaux pénètrent dans les eaux souterraines à partir de diverses sources naturelles, telles que l'altération chimique des roches et des sols, la végétation et les matières animales en décomposition et les retombées humides et sèches de particules atmosphériques (Driscoll et al., 1994, Karbassi et al., 2008). Cependant, certaines activités anthropiques peuvent apporter des quantités considérables dans les eaux. II s'agit entre autres de l'extraction minière, les piles, les matériaux ménagers jetables, les pesticides (Kumar et al., 2012 ; Zakhem and Hafez, 2015). Dans le département de Zouan-Hounien, l'extraction minière artisanale de l'or est l'une des sources majeures susceptibles de dégrader la qualité des ressources en eau souterraines. Pourtant, les eaux souterraines (puits et forages) sont les seuls moyens d'approvisionnement en eau des différentes localités du département. Les orpailleurs traitent le minerai et utilisent le mercure à proximité des points d'eau de consommation, augmentant les risques de pollution de ces ressources en eau. L'utilisation du mercure pour l'amalgamation de l'or, ainsi que les rejets d'effluents issus du lavage du minerai broyé, à proximité des points d'eau, sont susceptibles de détériorer la qualité des eaux par la mobilisation et la dispersion des éléments en trace dans l'environnement, notamment dans les eaux souterraines par infiltration (Bamba et al., 2013). Dès lors, l'évaluation des éléments traces métalliques dans les ressources en eaux souterraines, utilisées à des fins domestiques revêt une grande importance du point de vue de la santé humaine. L'objectif de cette étude est d'évaluer la qualité des ressources en eaux souterraines à travers le calcul des indices de pollution par les éléments traces métalliques, que sont: le facteur de contamination $(F C)$, le degré de contamination (Dc) et l'indice de pollution par les métaux lourds (HPI). 


\section{MATERIEL ET METHODES}

Présentation de la zone d'étude :Située à l'ouest de la Côte d'Ivoire, plus précisément dans le département de Zouan-Hounien, la zone d'étude est localisée entre les latitudes $06^{\circ} 50^{\prime}$ et $06^{\circ} 55^{\prime}$ Nord et les longitudes $08^{\circ} 05^{\prime}$ et $08^{\circ} 10^{\prime}$ Ouest (Figure 1). Elle est constituée de huit (8) villages riverains à la société minière d'Ity. $\mathrm{Ce}$ sont: Kouèpleu, Trogleu, Méantouo, Ouyatouo, Ity, Krozialé, Floleu et Biétouo. Ces villages abritent les acteurs principaux de l'exploitation minière artisanale et pour certains, les activités d'orpaillage. Cette zone d'étude est sous l'influence du climat de montagne, marqué par deux grandes saisons dont une saison pluvieuse et une saison sèche. La saison pluvieuse s'étend de Mai à Octobre et la saison sèche, quant à elle s'étend de Novembre à Mars (Brou et al., 2017). Les précipitations annuelles sont en moyenne de 1866 $\mathrm{mm}$, avec une température moyenne annuelle à ZouanHounien de $25,6^{\circ} \mathrm{C}$. Cette zone est caractérisée par des sols ferralitiques formés sur le substratum amphibolique (Dabin et al., 1960 ; Naho, 1988). Les formations géologiques de la zone d'étude sont constituées d'ortho-amphibolites, de schistes amphibolites, de tufs et quartzites à séricite et de rares passées de cornéennes et de skarns (Naho, 1988; Ettien, 2005).

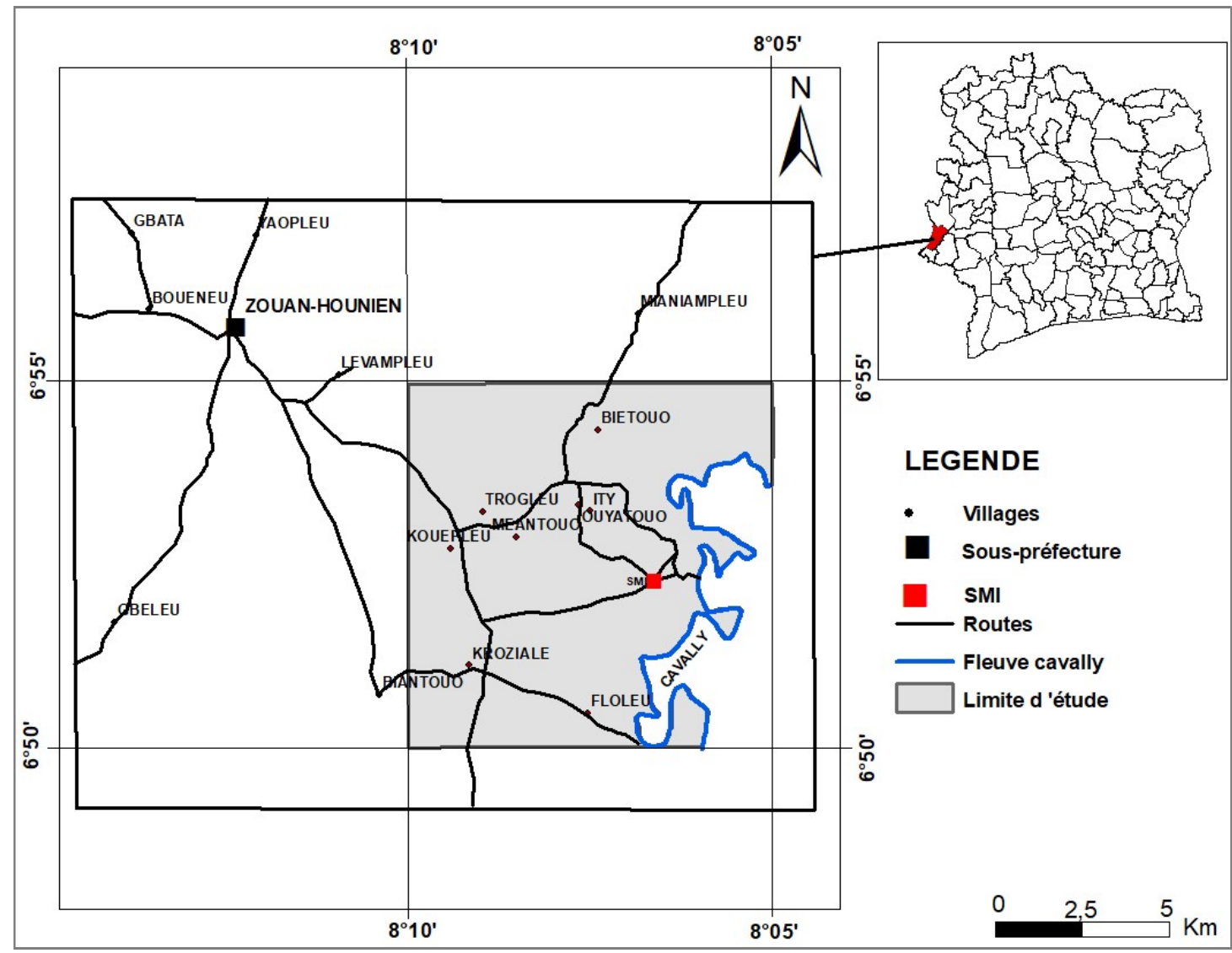

Figure 1 : Situation géographique de la zone d'étude

Campagne d'échantillonnage et analyse de laboratoire: Des échantillons d'eau ont été prélevés dans Vingt - trois (23) puits paysans et treize (13) forages lors de deux campagnes d'échantillonnage (Figure 2). La première campagne s'est déroulée dans le mois de mars, pendant la période sèche et la seconde dans le mois d'octobre, pendant la saison des pluies. Les échantillons d'eau de puits ont été prélevés à l'aide d'une puisette et recueillis dans des bouteilles en polyéthylène de $250 \mathrm{ml}$ de volume. Quant aux forages dotés d'une pompe à main, les eaux ont été prélevées directement à la pompe après avoir fait vider l'eau restée dans les baudruches. Avant le remplissage, les bouteilles ont été rincées plusieurs fois avec l'eau à analyser, remplies à ras bord et fermées hermétiquement afin d'éviter toute présence de bulles 
d'air. Les échantillons ont été acidifiés avec de l'acide nitrique $\left(\mathrm{HNO}_{3}\right) 6 \mathrm{M}$ ultrapur à $\mathrm{pH} \leq 2$, de sorte que les métaux dissous soient maintenus sous forme ionique, ce qui permettra leur détection par l'appareil de spectrométrie d'absorption atomique (SAA). Tous ces échantillons ont été conservés à basse température dans des glacières contenant des packs réfrigérants et acheminés au Laboratoire Central pour l'Hygiène Alimentaire et l'Agro-industrie (LCHAl) pour les différentes analyses. Les analyses de laboratoire ont porté uniquement sur les éléments traces métalliques ( $\mathrm{Hg}, \mathrm{As}, \mathrm{Cd}, \mathrm{Pb}$ et $\mathrm{Fe}$ ) par la méthode de Spectrométrie d'Absorption Atomique sans flamme pour le mercure selon la norme NF T90-113 (1986). Quant aux autres ETM, ils ont été dosés par la méthode Spectrométrique d'Absorption Atomique dans la flamme selon la norme NF T90-112 (1986) (AFNOR, 2008).

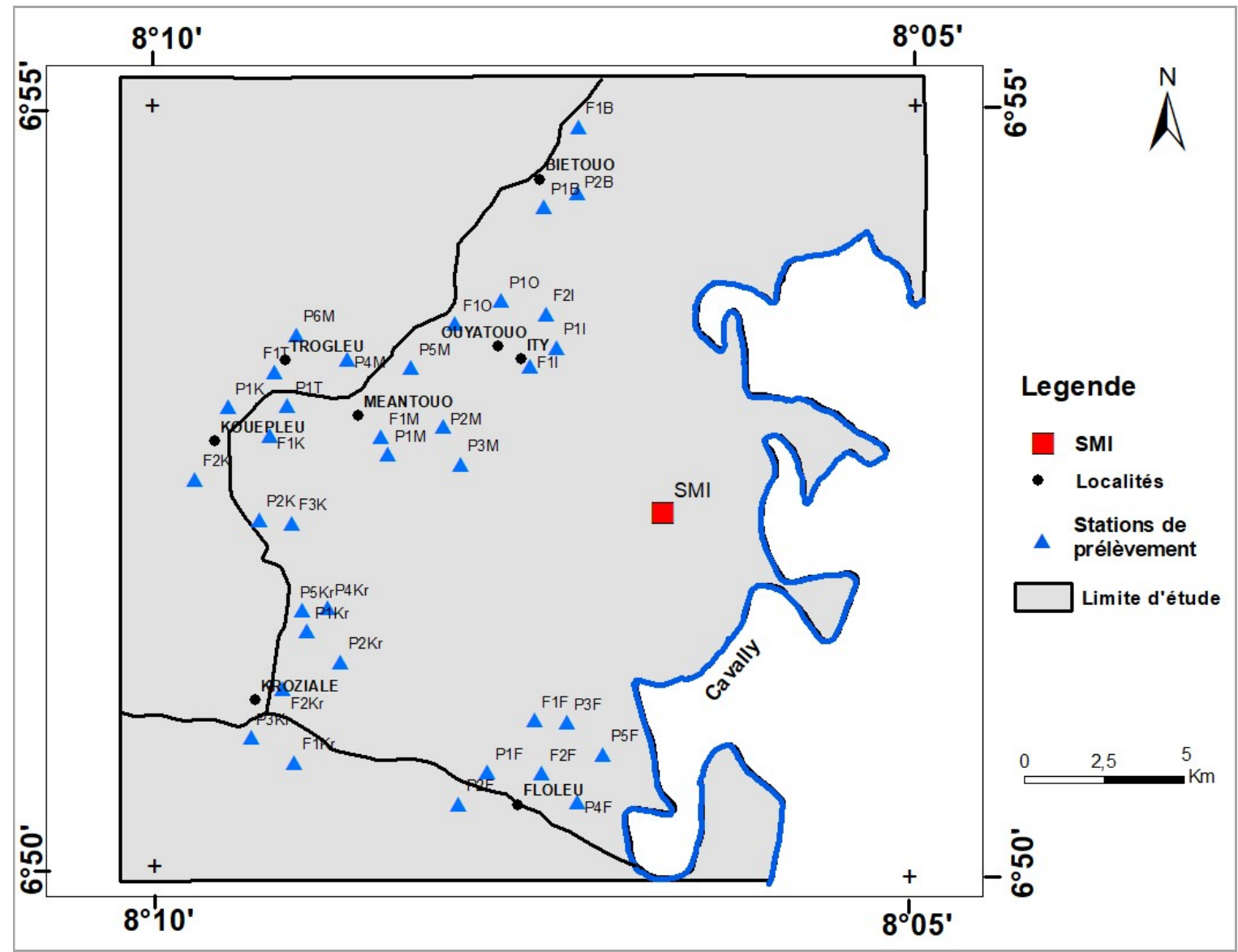

Figure 2 : Carte d'échantillonnage des eaux souterraines dans la zone d'étude

Méthode d'évaluation de la qualité des eaux par les ETM : Trois indices de pollution liés à la qualité des eaux de consommation ont été calculés. II s'agit du facteur de contamination (FC), du degré de contamination (Dc) et de l'indice de pollution des métaux lourds (HPI : heavy metal pollution index:).

Facteur de contamination (FC): Le facteur de contamination est un indice introduit par Hakanson (1980). C'est un outil simple et efficace pour surveiller la contamination du milieu par les éléments traces métalliques (Shen et al., 2019). II permet de mettre en évidence une contamination ou une absence de contamination du milieu par les ETM et donne le niveau de contamination. Le FC s'exprime par le rapport entre la concentration de l'élément mesurée dans le milieu et la concentration de référence (Equation 1). Dans le cadre de cette étude, la valeur guide de l'OMS (2017) pour les eaux de consommation, est prise comme valeur de référence. 


\section{$\mathrm{FC}=\frac{\text { Concentration de l'ETM mesurée dans l'eau }}{\text { Valeur guide de référence (OMS) }}$}

Cet indice est classé en quatre catégories selon Hakanson (1980):

- $\quad \mathrm{FC}<1=$ faible contamination ;

- $\quad 1<\mathrm{FC}<3=$ contamination modérée ;

- $\quad 3<\mathrm{FC}<6=$ contamination considérable ;

- $\quad F C>6=$. Très forte contamination.

$$
D c=\sum_{i=n}^{n} F c i
$$

Où Fciest le facteur de contamination du jième paramètre; et $\mathrm{n}$, la valeur normative de l'ETM. Le Dc est classé en trois catégories, à savoir: faible $(D c<1)$, moyen $(D c=1-3)$ et élevé $(D c>3)$ (Edet et Offiong 2003; Yari et Sobhanardakani, 2016; El-Hamid et Hegazy, 2018).

Indices de pollution des ETM (HPI): Le HPI est un indice proposé par Mohan et al. (1996). C'est un outil très utile pour évaluer la pollution globale des masses d'eau par les ETM (Prasad et Bose 2001; Edet et Offiong 2003; El-Hamid et Hegazy, 2017 ; Giri et Singh, 2019). Cette méthode est basée sur la qualité arithmétique pondérée et développée en deux étapes :

$$
\begin{gathered}
\mathrm{HPI}=\frac{\sum_{i=1}^{n} W i Q i}{\sum_{i=1}^{n} W i} \\
\mathrm{Qi}=\sum_{n=1}^{n} \frac{(M i-I i)}{(S i-I i)} * 100 \\
\mathrm{~W}_{1}=\mathrm{K} / \mathrm{MAC}
\end{gathered}
$$

Où Wi est la pondération du jième paramètre, Qi est l'indice du jième paramètre, Mi est la valeur surveillée de l'élément trace du iième paramètre; li est la valeur idéale du jième paramètre, Kest la constante de proportionnalité qui est égale à $1, \mathrm{Si}$ est la valeur standard du iième paramètre; (la norme adoptée est la limite de I'OMS (2017), $n$ est le nombre de paramètres MAC (en anglais maximum admissible concentration) : concentration maximale admissible relative à chaque
Degré de contamination (Dc): Le degré de contamination est utilisé comme référence pour évaluer le niveau de contamination des eaux par les métaux (Belkhiri et al., 2018). II ne prend pas en compte le niveau de pollution des eaux. Le degré de contamination a été calculé à partir de l'équation 2:

la première consiste à établir une échelle de notation pour chaque pondération donnant le paramètre sélectionné. La seconde, permet d'établir le paramètre de pollution sur lequel l'indice doit être basé (Singh et Kamal, 2016). Le système de notation est une valeur arbitraire comprise entre zéro et un et sa sélection dépend de l'importance des considérations de qualité individuelles de manière comparative ou peut être évaluée en rendant les valeurs inversement proportionnelles à la norme recommandée (Si) pour le paramètre correspondant (Prasad et Bose, 2001). Le calcul de HPI est donné par les équations 3, 4 et 5:

paramètre. La qualité de l'eau basée sur le HPI peut être classée en trois catégories : risque faible (HPI < $100)$, le risque de seuil $(\mathrm{HPI}=100)$ et risque élevé (> 100). La valeur critique de l'indice de pollution par les ETM est de 100 (Prasad et al., 2014). Pour des HPI supérieurs à 100, l'eau est impropre à la consommation (Aloueimine et al., 2017). Les valeurs standards qui servent au calcul de HPI sont présentées dans le tableau 1. 
Djade et al., J. Appl. Biosci. 2020 Evaluation du niveau de contamination des eaux souterraines par les éléments traces métalliques dans le département de Zouan-Hounien (Ouest de la Côte d'Ivoire).

Tableau 1 : Valeurs standards des ETM pour le calcul de HPI

\begin{tabular}{lllll}
\hline ETM & Wi & Si & li & MAC \\
\hline $\mathrm{Hg}$ & 1 & 2 & 1 & 1 \\
$\mathrm{~Pb}$ & 0,7 & 100 & 10 & 1,5 \\
$\mathrm{Cd}$ & 0,3 & 5 & 3 & 3 \\
$\mathrm{As}$ & 0,02 & 50 & 10 & 50 \\
$\mathrm{Fe}$ & 0,005 & 300 & 200 & 200 \\
\hline
\end{tabular}

\section{RESULTATS ET DISCUSSION}

Dynamique des ETM dans les eaux souterraines : Les concentrations des ETM dans les eaux de puits et de forages sont présentées dans le tableau 2. L'analyse de ce tableau montre que les concentrations moyennes de $\mathrm{Hg}, \mathrm{Pb}, \mathrm{Cd}$, As et $\mathrm{Fe}$ sont respectivement de $1,67,3,10,0,08,1,18$ et $2233,48 \mu \mathrm{g} . \mathrm{L}^{-1}$ dans les puits et de 1,76, 4,08, 0,08, 2,36 et 2427,94 $\mu \mathrm{g} \cdot \mathrm{L}^{-1}$ dans les forages. Les éléments sont abondants dans les eaux selon l'ordre: $\mathrm{Fe}>\mathrm{Pb}>\mathrm{Hg}>\mathrm{As}>\mathrm{Cd}$ Ces résultats révèlent que toutes les concentrations des ETM obtenues dans les puits et les forages, à l'exception de Fe sont conformes aux valeurs guide de l'OMS pour les eaux de consommation. Le fer est le métal le plus abondant dans les eaux. Les concentrations moyennes dans les puits et les forages pour ce dernier sont supérieures à la limite autorisée par l'OMS (2017) pour les eaux potables $\left(300 \mu \mathrm{g} \cdot \mathrm{L}^{-1}\right)$. Les fortes concentrations de fer observées dans les eaux souterraines, sont dues à la nature géologique de la zone. En effet, la géologie de la zone d'étude est constituée principalement d'amphiboles. Ce sont des roches ferromagnésiennes, dont l'altération va entrainer une accumulation du fer dans les sols, laissant place à des sols ferralitiques. La nature ferralitique des sols de la zone a été signalée par Dabin et al. (1960) et Naho (1988). Le fort lessivage de ces sols par les pluies, entraine une infiltration et une accumulation de cet élément dans les eaux souterraines. Des concentrations similaires de Fer ont été rapportées par Ahoussi et al. (2013) dans les eaux de puits de la souspréfecture de Biankouma, située dans l'ouest montagneux de la Côte d'Ivoire.

Tableau 2: Paramètres statistiques des ETM $\left(\mu \mathrm{g} \mathrm{L}^{-1}\right)$ dans les eaux souterraines et valeur guide de l'OMS (2017).

\begin{tabular}{lllllll}
\hline Matrices & $\begin{array}{l}\text { Paramètres } \\
\text { statistiques }\end{array}$ & Hg & Pb & Cd & As & Fe \\
& Min & 0,41 & 0,05 & 0,00 & $0,005,64$ & 775,19 \\
& Max & 4,63 & 6,56 & 0,18 & 0,60 & 3551,46 \\
Puits & Moy & 1,67 & 3,10 & 0,08 & 1,18 & 2233,48 \\
paysans & Ecart-type & 0,88 & $1,7556,50$ & 0,04 & 198,15 & 629,89 \\
& Cv (\%) & 52,69 & & 48,30 & & 28,20 \\
\cline { 2 - 6 } & Min & 0,55 & 0,12 & 0,00 & 0,14 & 1167,06 \\
& Max & 3,70 & 9,31 & 0,17 & 8,67 & 3944,44 \\
& Moy & 1,76 & 4,08 & 0,08 & 1,27 & 2427,94 \\
Forages & Ecart-type & 0,95 & 0,91 & 0,04 & 2,36 & 628,88 \\
& Cv (\%) & 58,89 & 22,36 & 52,67 & 185,85 & 25,90 \\
OMS (2017) & & $\mathbf{5}$ & $\mathbf{1 0}$ & $\mathbf{3}$ & $\mathbf{1 0}$ & $\mathbf{3 0 0}$ \\
\hline
\end{tabular}

Calcul des indices de pollution des ETM dans les puits et les forages: Les différentes valeurs moyennes des indices (FC, Dc et HPI) sont présentées dans tableau 3. L'analyse de ce tableau révèle que les eaux de puits sont faiblement contaminées par $\mathrm{Cd}$ $(0,03)$ et As $(0,01)$, modérément contaminées par $\mathrm{Hg}$ $(1,66)$ et $\mathrm{Pb}(2,06)$ et fortement contaminées par $\mathrm{Fe}$ $(11,17)$. Le degré de contamination (Dc), utilisé comme référence pour estimer l'intensité de la contamination des eaux, révèle un fort degré de contamination des eaux de puits. La valeur moyenne calculée est estimée à 14,93. Cette valeur largement supérieure à la valeur seuil de 3, pour l'ensemble des puits. Le HPI appliqué aux eaux afin d'évaluer leur niveau de pollution, révèle une faible pollution des eaux, avec une valeur moyenne de $67,58 \pm 38,48$. Cette valeur reste inférieure à 100 , définie comme valeur critique par Prasad et Bose (2001). Spécifiquement, les eaux dans 2 puits (P1Kr, $\mathrm{P} 1 \mathrm{I})$ sur $23(8,69 \%)$ se sont révélées fortement polluées. Ces valeurs sont respectivement de 209,26 et 
121,75, qui sont supérieures à 100. La qualité de ces eaux reste impropre à la consommation humaine, comme signalée par Aloueimine et al. (2017). Le HPI obtenu dans les puits P1F $(99,15)$ et P4F $(96,85)$ sont envoisinent la valeur de 100. Dans les forages, le FC de $\mathrm{Cd}$ et As sont inférieurs à 1, indiquant un faible niveau de contamination. Les eaux sont modérément contaminées par $\mathrm{Hg}(1,76)$ et $\mathrm{Pb}(2,72)$ avec des FC compris entre 1 et 3 . Pour Fe, le FC est de 12,14, valeur supérieure à 6 . Ce qui montre que les eaux sont fortement contaminées par ce dernier. Ces facteurs conduisent à un fort degré de contamination des eaux de forages, d'une valeur de 16,67, largement supérieure à la valeur seuil de 3 . Malgré le fort degré de contamination des eaux, elles restent en moyenne faiblement polluées avec une valeur moyenne de HPI inférieure à $100(73,92 \pm 40,16)$. Néanmoins, 2 forages sur 13 (F2Kr et $\mathrm{F} 1 \mathrm{l})$ ont enregistré un $\mathrm{HPI}$ supérieur à la valeur seuil, soit un taux de $15,38 \%$. Les valeurs enregistrées dans le forage $\mathrm{F} 2 \mathrm{Kr}$ et $\mathrm{F} 1 \mathrm{l}$ sont respectivement de 140,98 et 163,16. Le fort degré de contamination des eaux de puits et de forages est dû aux valeurs élevées du fer enregistrées à toutes les stations. Le FC du Fe calculé dans toutes les eaux a révélé que les eaux sont fortement contaminées. Or le Dc est la résultante de tous les $\mathrm{FC}$, ce qui a entrainé un fort degré de contamination des eaux de puits et forages. Des travaux similaires ont été rapportés par Batabyal et Chakraborty (2015) dans les eaux souterraines destinées à la consommation dans le nord du district Bardhaman, situé à l'ouest de Bengale en Inde. Ces auteurs ont révélé une mauvaise qualité des eaux souterraines à travers des valeurs élevées d'indices de qualité des eaux. Ce qui a été attribué aux fortes concentrations de fer enregistrées dans les eaux (0.05 - 14 mg. $\mathrm{L}^{-1}$ et $\left.0.11-16.8 \mathrm{mg} . \mathrm{L}^{-1}\right)$. Les différentes stations ainsi que les pourcentages en fonction du degré de contamination et le niveau de pollution sont reportés dans le tableau 4. II ressort de ce tableau que dans les puits, la totalité des ouvrages $(100 \%)$ a un degré de contamination élevé. Pour le HPI, 21 puits $(91,30 \%)$ ont enregistré un faible niveau de pollution. En revanche, 2 puits soit $8,70 \%$ ont enregistré une forte pollution. Tout comme dans les puits, tous les forages $(100 \%)$ ont des eaux avec un fort degré de contamination. Avec les valeurs de HPI calculées dans les eaux, 11 forages $(84,62 \%)$ se sont révélés faiblement pollués. Inversement, 2 forages $(15,38 \%)$ se sont montrés fortement pollués.

Corrélation entre les ETM et HPI : Le coefficient de corrélation de Pearson a été utilisé pour mettre en évidence l'implication de $\mathrm{Hg}, \mathrm{Pb}, \mathrm{Cd}$, As et $\mathrm{Fe}$ dans la pollution des eaux de consommation, à travers une matrice de corrélation (Tableau 5). Le HPI, qui est le principal indice d'évaluation de la pollution des eaux par les ETM, est le seul indice incorporé dans la matrice de corrélation pour examiner le rôle que joue chaque élément dans la toxicité des eaux. Le tableau montre que $\mathrm{Hg}$ est le seul élément fortement corrélé au HPI, avec un coefficient de corrélation de Pearson de 0,95. Certains ETM pris deux à deux expriment d'importantes liaisons. Fe est fortement lié au $\mathrm{Cd}(0,82)$ et à un degré moindre avec $\mathrm{Pb}(0,74)$. Cd et $\mathrm{Pb}$ sont fortement corrélés $(0,80)$. La forte liaison observée entre $\mathrm{Fe}, \mathrm{Pb}$ et $\mathrm{Cd}$, suggère une origine commune de ces trois éléments. En effet, la géologie de la zone d'étude est constituée de roches amphibolites, dont l'altération conduit aux sols ferralitiques. Cette particularité confère au fer une origine naturelle terrigène. Ainsi, son affinité avec $\mathrm{Cd}$ et $\mathrm{Pb}$ suggère que la présence de ces deux ETM dans les eaux est d'origine naturelle. Selon Lu et al. (2010) et Saeedi et al. (2012), les fortes corrélations entre certains métaux lourds indiquent les mêmes sources d'intrants et un comportement géochimique similaire. La forte corrélation obtenue entre $\mathrm{Hg}$ et $\mathrm{HPI}$, et le manque de liaison avec les autres éléments montre que la présence de ces paramètres dans les eaux n'est pas régie par le même phénomène. Cela suggère une autre origine du mercure, autre que l'origine naturelle terrigène. Elle est attribuable aux activités d'orpaillage, qui utilisent cette substance dans l'amalgamation de l'or. De plus, l'orpaillage est une activité ancienne dans la zone. Selon Ettien (2010), d'intenses activités d'orpaillage existaient depuis les années 1940 et 1950, ce qui a pu favoriser une accumulation de mercure dans le sol. La conversion de tous ces espaces en des villages aujourd'hui, peut expliquer les fortes valeurs de mercure obtenues dans les eaux, plus particulièrement dans les forages. Car selon plusieurs travaux, le mercure peut persister dans l'environnement pendant plusieurs décennies (Oswald et al., 2014 ; Hsu-Kim et al., 2018). En effet, le mercure comprend 40 isotopes dont le nombre de masse varie de 171 à 210 (Meierfrankenfeld et Thoennessen, 2009). Selon ces auteurs, la durée de vie de ces isotopes varie entre 5,5 minutes $\left({ }^{205} \mathrm{Hg}\right)$ et 444 ans $\left({ }^{194} \mathrm{Hg}\right)$. Vu que le dosage du mercure dans le cadre de cette étude n'a porté que sur le mercure total et qu'aucune étude d'isotopie n'a été effectuée, il est probable que la forme la plus stable du mercure soit comprise dans ce mercure total. Ainsi, la contamination des eaux souterraines est attribuable d'une part à une 
contamination due à l'orpaillage ancien. Ces travaux sont conformité avec ceux de Boudou et al. (2006); Guedron et al. (2006) et Grimaldi et al. (2008), qui ont rapporté que les sols amazoniens et guyanais renfermaient d'importantes quantités de mercure à cause de l'orpaillage ancien. D'autre part, la contamination atmosphérique constitue une source de présence du mercure dans les eaux souterraines. En effet, le mercure utilisé pour amalgamer l'or a un caractère volatile (Veiga et Baker, 2004). Le procédé consiste à dissoudre l'or dans le mercure et former avec lui un amalgame (mélange de mercure et de paillette d'or). Lors de la fusion de l'amalgame, ce dernier s'évapore dans l'atmosphère pour se déposer sur le couvert végétal, le toit des maisons, le sol, les ressources en eau et les puits non couverts, les terres arables (Grimaldi et al., 2015 ; Mallongi et al., 2017 ; Posthumus, 2019). Les fortes précipitations enregistrées dans la zone augmentent la mobilité du mercure en améliorant ses transferts verticaux et latéraux, ce qui accroit ses concentrations dans l'eau. La contamination atmosphérique des sols et des ressources en eau par le mercure a été mise en évidence par plusieurs auteurs (Barringer et al., 2012 ; Driscoll et al., 2013 ; Esdaile et Chalker, 2018).
Sur des sites d'orpaillage en Tanzanie et au Zimbabwe, Straaten (2000) a rapporté une contamination des eaux souterraines par les dépôts atmosphériques du mercure issu du traitement du minerai. Selon ce dernier, pour $1 \mathrm{~g}$ d'or produit, $1,2 \mathrm{~g}$ à $1,5 \mathrm{~g}$ de mercure sont perdus dans l'atmosphère dans ces pays. La pollution des eaux échantillonnées est gouvernée par deux principales sources, à savoir la lithologie de la zone et les activités minières. La corrélation entre $\mathrm{Hg}$ et $\mathrm{HPI}$ sont similaires à ceux de Kwaya et al. (2019), dans les eaux souterraines de la ville de Maru et ses environs, au nord du Nigéria. Travaux dans lesquels, le $\mathrm{Cd}$ est l'élément le mieux corrélé au HPI et dont les concentrations élevées ont été attribuées aux activités minières. Cependant, les eaux de cette étude restent fortement polluées par rapport à l'actuelle étude (HPI = $3129,79)$. Les valeurs de HPI, rapportées par Yankey et al (2013) dans les eaux de forages de la zone minière de Tarkwa sont inférieures à celle de la présente étude $(\mathrm{HPI}=53,70)$. Sur la base des résultats obtenus, le HPI est l'indice idéal pour évaluer la qualité des eaux par les ETM. II est précis dans le calcul de la pollution et prend en compte le poids de chaque élément (Rezaverdinejad et Rahimi, 2017). De plus, les valeurs de HPI étaient en parfait accord avec les concentrations d'ETM obtenues dans les eaux.

Tableau 3 : Indices de contamination et de pollution par les ETM (HPI et Dc) dans les eaux de forages et puits

\begin{tabular}{llll}
\hline & Paramètres & Dc & HPI \\
statistiques & & \\
\hline \multirow{4}{*}{ Puits } & Min & 0,87 & 38,48 \\
& Max & 17,57 & 209,25 \\
& Moy & 9,91 & 67,58 \\
& Ecart-type & 3,87 & 38,48 \\
Forages & Cv (\%) & 39,10 & 56,94 \\
& Min & 4,20 & 34,50 \\
& Max & 23,75 & 163,16 \\
& Moy & 11,68 & 73,92 \\
& Ecart-type & 4,79 & 40,17 \\
& Cv (\%) & 41,04 & 54,34 \\
\hline
\end{tabular}


Tableau 4 : Classification de la qualité des eaux souterraines de la zone d'étude selon les catégories de degré de contamination et d'indices de pollution.

\begin{tabular}{|c|c|c|c|c|c|c|}
\hline Matrices & Indices & Classification & $\begin{array}{l}\text { Degré } \\
\text { contamination et } \\
\text { de pollution }\end{array}$ & $\begin{array}{l}\text { Nombre } \\
\text { d'échantillons }\end{array}$ & \% d'échantillons & Noms des stations \\
\hline \multirow{8}{*}{$\begin{array}{l}\text { Puits } \\
\text { paysans } \\
n=23\end{array}$} & \multirow{4}{*}{ Dc } & $<1$ & Faible & 01 & 4,35 & P4Kr \\
\hline & & $1-3$ & Modérée & 01 & 4,35 & $\mathrm{P} 5 \mathrm{Kr}$ \\
\hline & & $>3$ & Elevée & 21 & 91,30 & P1F, P2F, P3F, P4F, P5F, P1Kr, P2Kr, \\
\hline & & & & & & $\begin{array}{l}\text { P3Kr, P1K, P2K, P1I, P1T, P1M, P2M, P3M, } \\
\text { P5M, P6M, P4M, P1O, P1B, P2B }\end{array}$ \\
\hline & \multirow{4}{*}{ HPI } & $<100$ & Faible & 19 & 82,60 & P2F, P2F, P3F, P5F, P2Kr, P3Kr, P4Kr, \\
\hline & & & & & & $\begin{array}{l}\text { P5Kr, P1K, P2K, P1T, P1M, P2M, P3M, } \\
\text { P5M, P6M, P4M, P1O, P1B, P2B }\end{array}$ \\
\hline & & $=100$ & Modérée & 02 & 8,70 & P1F, P4F \\
\hline & & $>100$ & Elevée & 02 & 8,70 & P1Kr, P1I, \\
\hline \multirow{7}{*}{$\begin{array}{l}\text { Forages } \\
n=13\end{array}$} & \multirow{4}{*}{ Dc } & $<1$ & Faible & 00 & 00,00 & - \\
\hline & & $1-3$ & Modérée & 00 & 00,00 & - \\
\hline & & $>3$ & Elevée & 13 & 100,00 & F1F, F2F, F1Kr, F2Kr, F1K, F2K, F3K, F1T, \\
\hline & & & & & & F1M, F10, F1I, F2I, F1B \\
\hline & \multirow{3}{*}{ HPI } & $<100$ & Faible & 11 & 84,61 & F1F, F1Kr, F1K, F2K, F3K, F1T, F10, F2I, \\
\hline & & $=100$ & Modérée & 00 & 00,00 & - \\
\hline & & $>100$ & Elevée & 02 & 15,38 & $\mathrm{~F} 2 \mathrm{Kr}, \mathrm{F} 1 \mathrm{l}$ \\
\hline
\end{tabular}

$\mathrm{P}$ : puits, $\mathrm{F}$ : forages ; F : Floleu, Kr : Krozialé, K : Kouèpleu, T : Trogleu, M : Méantouo, O : Ouyatouo, I : Ity, B : Biétouo.

Tableau 5 : Matrice de corrélation entre les ETM et les indices de pollution (HPI)

\begin{tabular}{|c|c|c|c|c|c|c|}
\hline & $\mathrm{Hg}$ & $\mathrm{Pb}$ & $C d$ & As & $\mathrm{Fe}$ & HPI \\
\hline $\mathrm{Hg}$ & 1 & & & & & \\
\hline $\mathrm{Pb}$ & $-0,09$ & 1 & & & & \\
\hline $\mathrm{Cd}$ & $-0,19$ & 0,80 & 1 & & & \\
\hline As & 0,04 & 0,44 & 0,30 & 1 & & \\
\hline $\mathrm{Fe}$ & $-0,17$ & 0,73 & 0,82 & 0,36 & 1 & \\
\hline HPI & 0,95 & $-0,10$ & $-0,18$ & 0,09 & $-0,14$ & 1 \\
\hline
\end{tabular}




\section{CONCLUSION}

L'étude de la qualité des eaux dans certaines localités du département de Zouan-Hounien, montre que les eaux souterraines sont riches en fer. Les concentrations moyennes de cet élément sont supérieures à la valeur guide recommandée par l'OMS. Le $\mathrm{Hg}$, le $\mathrm{Pb}$, le $\mathrm{Cd}$ et l'As enregistrent des concentrations conformes aux valeurs recommandées pour les eaux de consommation. L'indice de pollution des éléments en trace appliqué aux eaux révèle que les

\section{REFERENCES}

Ahoussi K, Koffi YB, Kouassi AM, Soro G, Biemi J, 2013. Étude hydrochimique et microbiologique des eaux de source de l'ouest montagneux, Cote d'Ivoire : Cas du village de Mangouin Yrongouin (souspréfecture de Biankouman). J. Appl. Biosci. 63: 4703 - 4719.

Aloueimine BB, Kankou MO, Belghyti D, 2017. An indexing approach for the assessment of heavy metals in drinking water produced by Mauritanian water treatment plant. Scientific Study \& Research 18: 319 - 328.

AFNOR (2008), NF T90-112. 1986. Essais des eaux Dosage de dix éléments métalliques $(\mathrm{Cr}, \mathrm{Mn}$, $\mathrm{Fe}, \mathrm{Co}, \mathrm{Ni}, \mathrm{Cu}, \mathrm{Zn}, \mathrm{Ag}, \mathrm{Cd}, \mathrm{Pb}$ ) par spectrométrie d'absorption atomique dans la flamme - Méthode par dosage direct (NF T90112 Septembre 1986). Code Sandre : 119, 1p. http://mdm.sandre.eaufrance.fr/id/methode/11 9/html

AFNOR (2008), NF T90-113. 1986. Essais des eaux Dosage du mercure total par spectrométrie d'absorption atomique sans flamme - Méthode après minéralisation au permanganateperoxodisulfate (NF T90-113-2 Septembre 1986). Code Sandre : 122, 1p. http://mdm.sandre.eaufrance.fr/id/methode/12 2/html

Bamba O, Pélédé S, Sako A, Kagambéga N, Miningou1 MYW, 2013. Impact de l'artisanat minier sur les sols d'un environnement agricole aménagé au Burkina Faso. Journal des sciences 13: 1 11.

Barringer JL, Szabo Z, Reilly PA, 2013. Occurrence and Mobility of Mercury in Groundwater, Chapter 5. Licence In Tech, pp: 116 - 150.

Batabyal A.K. et Chakraborty S, 2015. Hydrogeochemistry and Water Quality Index in the Assessment of Groundwater Quality for Drinking Uses. Water Environ. Res, 87 : 607 - eaux sont dans l'ensemble de bonne qualité, hormis deux puits et deux forages. L'orpaillage est la principale source d'émission du mercure dans les eaux. En revanche, le $\mathrm{Fe}$, le $\mathrm{Pb}$, le $\mathrm{Cd}$ et l'As sont d'origine naturelle. Ils proviennent de la géochimie de la zone. Malgré la faible pollution des eaux par les ETM, la présence de ces éléments dans les eaux peut poser des problèmes de santé pour la population.

\section{Doi: $\quad 10.2175 / 106143015$ X14212658613956.}

Belkhiri L, Tiri A, Mouni L 2018. Assessment of Heavy Metals Contamination in Groundwater: A Case Study of the South of Setif Area, East Algeria. IntechOpen, chapter 2, pp: 17-31. Doi: 10.5772/intechopen.75734.

Boudou A, Maury-Brachet R, Durrieu G, Coquery M, Dauta C, 2006. Chercheurs d'or et contamination par le mercure des systèmes aquatiques continentaux de Guyane - Risques à l'égard des populations humaines. Hydroécol. Appl. 15: 1-18. Doi: 10.1051/hydro:2006007

Brou LA, Kouassi KL, Konan KS, Kouadio ZA, Dongo TF, Konan KF, Kamagaté B, 2017. Modélisation Pluie-Débit à l'aide des Réseaux de Neurones Artificiels Multicouches sur le Bassin Versant du Fleuve Cavally à la station d'Ity (Zouan-Hounien, Côte d'Ivoire). EauSociété-Climat (ESC 2017). Vol 5, 7p.

Dabin B, Leneuf N, Riou G, 1960. Carte pédologique de la Côte d'Ivoire au 1-2.000.000. Notice explicative, ORSTOM. 39p.

Driscoll CT, James JK, Akeiverfeldt, 1994. 13. Trace metals speciation and cycling in Biogeochemistry of small catchments: a tool for environmental research Edited by Moldan B, Cemy J (eds). pp. 299-322.

Driscoll CT, Mason RP, Chan HM, Daniel JJ, Pirrone N, 2013. Mercury as a Global Pollutant: Sources, Pathways, and Effects. Environmental Science \& Technology 47: 4967-4983. Doi.org/10.1021/es305071v

Edet $\mathrm{AE}$ et Offiong $\mathrm{OE}, 2003$. Evaluation of water quality pollution indices for heavy metal contamination monitoring. A study case from Akpabuyo-Odukpani area, Lower Cross River 
Basin (Southeastern Nigeria). Geojournal 57: 295-304.

El- Hamid HTA et Hegazy TA, 2017. Evaluation of Water Quality Pollution Indices for Groundwater Resources of New Damietta, Egypt. MOJ Eco Environ Sci. 2: 1-5. Doi: 10.15406/mojes.2017.02.00045.

Esdaile LJ et Chalker JM, 2018. The Mercury Problem in Artisanal and Small-Scale Gold Mining. Chem. Eur. J. 24: 6905-6916. Doi: 10.1002/chem.201704840.

Ettien DZ, 2005. Étude d'évaluation de l'impact des exploitations minières sur l'environnement et les populations en Afrique occidentale : Cas de la mine d'or d'lty dans la région semimontagneuse de l'Ouest de la Côte d'Ivoire. Apport du Système d'Informations Géographiques (S.I.G) et de la télédétection. Thèse Unique de Doctorat, Université de Cocody, Abidjan, $178 \mathrm{p}$.

Ettien DZ, 2010. Exploitation industrielle des gisements d'or et dynamique spatiale du terroir d'Ity dans l'Ouest de la Côte d'Ivoire. Une étude à base de la télédétection. RGLL 08 : 1-15.

Giri S, et Singh AK, 2019. Assessment of metal pollution in groundwater using a novel multivariate metal pollution index in the mining areas of the Singhbhum copper belt. Environmental Earth Sciences 78: 1-11. doi:10.1007/s12665-019-8200-9

Grimaldi C, Grimaldi M, Guedron S, 2008. Mercury distribution in tropical soil profiles related to origin of mercury and soil processes: Science of The Total Environment 401:121-129. DOI: 10.1016/i.scitotenv.2008.04.001.

Grimaldi M, Guédron S, Grimaldi C, 2015. 9 Impact of Gold Mining on Mercury

Contamination and Soil Degradation in Amazonian Ecosystems of French Guiana. (c) CAB International, pp : 95 - 111.

Guédron S, Spadini L, Grimaldi C, Chauvel C, Grimaldi $M, 2006$. Weathering versus atmospheric contributions to mercury concentrations in French Guiana soils. Applied Geochemistry $21: 2010-2022$

Hakanson L, 1980. Ecological risk index for aquatic pollution control, a sedimetological approach. Water Research 14 : 975-1001.

Hsu-Kim H, Eckley CS, Acha D, Feng X, Gilmour CC, Jonsson S Mitchell CPJ, 2018. Challenges and opportunities for managing aquatic mercury pollution in altered landscapes. Ambio 47 : 141-169. Doi 10.1007/s13280-017-1006-7.

Karbassi AR, Monavari .M, Nabi BGR, Nouri J, Nematpour K, 2008. Metal pollution assessment of sediment and water inthe Shur River. Environ Monit Assess 147: 107-116. Doi: 10.1007/s10661-007-0102-8.

Kumar PJS, Delson PD, Babu PT, 2012. Appraisal of Heavy Metals in Groundwater in Chennai City Using a HPI Model. Bull Environ Contam Toxicol. 89 : 793-798. doi. 10.1007/s00128012-0794-5.

Kwaya MY, Hamidu H, Mohammed Al, Abdulmumini YN, Grema IHAHM, Dauda M., Halilu F. B., Kana A. M. 2019. Heavy Metals Pollution Indices and Multivariate Statistical Evaluation of Groundwater Quality of Maru town and environs. Journal of Materials and Environmental Sciences 10: 32-44.

Lu X, Wang L, Li LY, Lei K, Huang L, Kang D, 2010. Multivariate statistical analysis of heavy metals in street dust of Baoji, NW China. Journal of Hazardous Materials, J Hazard Mater 173: 744-749. Doi. 10.1016/j.jhazmat.2009.09.001.

Mallongi A, Irwan, Rantetampang AL, 2017. Assessing the Mercury Hazard Risks among Communities and Gold Miners in Artisanal Buladu Gold Mine, Indonesia. Asian Journal of Scientific Research 10:316-322. Doi.10.3923/ajsr.2017.316.322.

Meierfrankenfeld D, et Thoennessen M, 2009. Discovery of the Mercury Isotopes. Preprint submitted to atomic data and nuclear data tables. 17p.

Mohan S. V., Nithila P., and Reddy S. J. 1996. Estimation of heavy metals in drinking water and development of heavy metal pollution index. J. Environ. Sci. Health 31A: 283-289. ICA no. M-3951.

Naho J, 1988. Cycle supergène de l'or en milieu ferralitique. Exemple du gisement d'Ity en Côte d'Ivoire. Thèse de Doctorat ès Géosciences et Matière Première, INPL, Nancy, 132p.

OMS (Organisation Mondiale de la Santé), 2017. Guidelines for Drinking-water Quality. Fourth edition incorporating the first Addendum, 631 p.

Oswald CJ, Heyes A, Branfireun BA, 2014. Fate and transport of ambient mercury and applied mercury isotope in terrestrial upland soils: Insights from the METAALICUS watershed. 
Environmental Science and Technology 48: 1023-1031. Doi : 10.1021/es404260f.

Posthumus EDJ, 2019. L'impact de l'extraction minière artisanale et à petite échelle de l'or sur la santé humaine et l'environnement au Burkina Faso. World Waternet, $8 p$. https://www.wereldwaternet.nl/en/latest-news /2019/october/the-impact-of-artisanal-andsmall-scale-gold-mining-on-human-health-andthe-environment-in-burkina-fasol. Conulté le 15 février 2020.

Prasad B, et Bose JM, 2001. Evaluation of the heavy metal pollution index for surface and spring water near a limestone mining area of the lower Himalayas. Environ Geol. 41: 183-188.

Prasad B, Kumari P, Bano S, Kumari S, 2014. Ground water quality evaluation near mining area and development of heavy metal pollution index. Appl Water Sci. 4: 11-17. Doi 10.1007/s13201-013-0126-X.

Rezaverdinejad V, et Rahimi M, 2017. Seasonal Assessment of Nitrate, Nitrite, and Heavy Metals Pollution in Groundwater of Ardabil Aquifer, Iran. Pol. J. Environ. Stud. 26: 22672276. Doi: 10.15244/pjoes/69943.

Saeedi M, Li LY, Salmanzadeh M, 2012. Heavy metals and polycyclic aromatic hydrocarbons: Pollution and ecological risk assessment in street dust of Tehran. Journal of Hazardous Materials $\quad 15:$ 9-17. Doi.org/10.1016/j.jhazmat.2012.04.047.

Shen F, Mao L, Sun R, Du J, Tan Z, Ding M, 2019. Contamination evaluation and Source Identification of Heavy Metals in the Sediments from the Lishui River Watershed, Southern China. International Journal of Environmental
Research and Public Health 16: 1 - 14. Doi: 10.3390/ijerph16030336.

Singh G, et Kamal RK, 2016. Heavy metal contamination and its indexing approach for groundwater of Goa mining region, India. Appl Water Sci. 7: 1479-1485. Doi:10.1007/s13201-016-0430-3.

Veiga MM, et Baker RF, 2004. Protocols for Environmental and Health Assessment of Mercury Released by artisanal and small-scall gold miners. Vienna, Austria: GEF/UNDP/UNIDO. 289p.

Vodela JK, Renden JA, Lenz SD, McElhenney WH, Kemppainen BW, 1997. Drinking water contaminants (arsenic, cadmium, lead, benzene, and trichloroethylene). Interaction of contaminants with nutritional status on general performance and immune function in broiler chickens. Poult Sci. 76: 1474-1492. Doi:10.1093/ps/76.11.1474.

Yankey RK, Fianko JR, Osae S, Ahialey EK, Duncan $\mathrm{AE}$, Essuman DK, 2013. Evaluation of heavy metal pollution index of groundwater in the Tarkwa mining area, Ghana. Elixir Pollution $54: 12663-12667$.

Yari AR et Sobhanardakani S, 2016. Water Quality Assessment of Ground water Resources in Qaleeh Shahin Plain Based on $\mathrm{Cd}$ and $\mathrm{HEl}$. International Archives of Health Sciences, 3 : 101-106.

Zakhem BA, et Hafez R, 2015. Heavy metal pollution index for groundwater quality assessment in Damascus Oasis, Syria. Environ Earth Sci. 73: 6591-6600. Doi: 10.1007/s12665-014$\underline{3882-5}$ 